\title{
LA AGENDA PSICOSOCIAL EN PERÚ: AUTOPERCEPCIÓN DE LOGROS, REPRESENTACIÓN DE EFICACIA SOCIAL Y RESPONSABILIDAD SOCIAL
}

\author{
Carlos Arenas I1'., Victor Montero L. \\ Universidad Nacional Mayor de San Marcos, Lima, Perú \\ (RECiBido El 22/09/2009, ACEPTADo EL 23/11/ 2009)
}

\begin{abstract}
RESUMEN
El propósito principal es evidenciar el papel que la psicología social desempeña en respuesta a las demandas sociales, identificar la autopercepción de los logros que los propios psicólogos sociales tienen de su profesión, contrastando ésta con la evidencia que las representaciones sociales de la eficacia que objetivamente se encuentran presentes y enmarcando todo este conjunto de autopercepciones y representaciones sociales en el ámbito de la responsabilidad social. Usando la metodología cualitativa (grupos focales, entrevistas, investigación participativa) se estudian el conjunto de percepciones y representaciones desde la responsabilidad social que le compete a la disciplina psicológica social. El cómo se percibe y representa la eficacia de su quehacer psicológico-social y cuál es la relación con el nivel de representación social que ellos han ido construyendo en el decurso de su formación académico-profesional. Los resultados esperados son demostrar la efectividad de la aplicación de la psicología social en las políticas y programas sociales frente a los problemas sociales prioritarios en el Perú, es decir la agenda y construir indicadores que faciliten el conocimiento de la efectividad de dichos programas en aspectos tales como los formativos, profesionales e investigativos y teóricos desde la propia perspectiva de los actores, los psicólogos sociales, en las diversas regiones del país.
\end{abstract}

Palabras clave: agenda psicosocial, autopercepción, representación social, responsabilidad social.

\section{ABSTRACT}

THE PSYCHO-SOCIAL AGENDA IN PERU: AUTOPERCEPTION OF ACHIEVEMENTS, REPRESENTATION OF SOCIAL EFFECTIVENESS AND SOCIAL RESPONSIBILITY

The main purpose is to highlight the role of social psychology in response to social demands, identify the self-perception of the achievements that own social psychologists have their profession, contrasting it with the evidence that social representations of effectiveness than objectively are present and framing this set of autopercepciones and social representations in the area of responsibility social. Using qualitative methodology (focus groups, interviews) (participatory research) explores the set of perceptions and representations from the social responsibility that you are responsible discipline psychologicosocial. How are perceived and

1 Docente Principal de la Facultad de Psicología, UNMSM. E-mail: postgradopsi@hotmail.com 
represent the effectiveness of its work psychológicosocia and what is the relationship with the level of social representation they have been built in the course of their training académicoprofesional. The expected results are to demonstrate the effectiveness of the implementation of the social psychology in policies and social programmes versus priority social problems in Peru, i.e. the agenda and build indicators to facilite the effectiveness if such knowledge programmes in areas such as training, research and professional and theorists from the perspective of actors, social psychologists, in the various regions of the country.

Keywords: psychosocial, self-awareness, social representation, psychosocial agenda, social responsibility.

\section{INTRODUCCIÓN}

La creencia de que la aportación de la psicología social (Zimbardo, Philip y Clemente, 1992) es que permite entender que la Psicología se legitima en la medida en que ayuda a mejorar la calidad de vida humana, a mejorar las condiciones materiales de existencia, a hacer consciente a las personas de que esas condiciones son fruto de lo humano y pueden ser cambiadas. Del mismo modo, la psicología social tiene el poder para afrontar con alguna garantía la transformación de algunos extremos del mundo real, muy probablemente actuando sobre el mundo de las percepciones, de las creencias y de las representaciones subjetivas de la naturaleza social. Martín Baró (1982) (El Salvador), Amalio Blanco (2002) (España) y Maritza Montero (1984) (Venezuela) han resaltado el aporte de la psicología social para desalinear explicaciones que deforman la realidad como las referentes a la pobreza, que lejos de ser un fenómeno natural responde a una estructura social injusta y corrupta; igualmente, aportan para comprender diversos fenómenos asociados a la identidad nacional, resaltando conceptos como de motivación de logro y de afiliación, experiencia de logro, recuperación crítica de la historia, autoestima, esperanza y otros. Como sustenta Páez (2004), una psicología social que surge y se desarrolla en respuesta a la problemática de contextos geográficos diferentes, es una interdisciplina en rigor capaz de potenciar los recursos y la cooperación de subjetividades, grupos y organizaciones, tendiente a la constitución de redes, porque cuenta con metodologías y técnicas participativas orientadas a un objetivo de transformación social (Adamson, 1999).

Existe un paralelismo, a veces conflictivo, de las teorías y la producción académica universitaria, con las intervenciones psicosociales en problemas sociales y de salud pública, ello nos llevó a preguntarnos cuál es la distancia entre ambos, y quizá más importante que ello, y desde los propios actores: los psicólogos sociales, cuáles son sus autopercepciones de eficacia, teniendo como marco referencial el constructo relacionado con la responsabilidad social.

Rappaport (1877) planteó considerarla como una perspectiva orientada a la búsqueda de nuevos paradigmas o formas de comprender y actuar, las cuales debían considerar tres elementos importantes: a) la relatividad cultural; b) la diversidad humana, el derecho de la gente a acceder a los recursos de la sociedad a elegir sus metas y estilo de vida y c) la ecología o ajuste entre personas y ambiente en el que destaca el social como un determinante del bienestar humano, del mismo modo autores como Mead (1965) o Vigotsky (s/f), vincularon los procesos cognitivos a la producción social de significados. Solo así es posible un estudio de la mente en el que no queden excluidos los procesos simbólicos. 
Progresivamente, se han ido mezclando tradiciones académicas y prácticas sociales, cuyo eje aglutinador han sido los problemas sociales.

Existe una demanda teórico-técnica a la psicología social en torno a los problemas sociales existentes. Y es desde allí donde han surgido o se ha expresado el aporte de distintas categorías psicosociales (Morales, G. 1998).

Según Moscovici (1965) y Martín Baró (1982), entre otros psicólogos sociales, afirman que hay necesidad de revisar y teorizar sobre las prácticas sociales y los problemas que se derivan de ella. Ellos apelan a un desafío descriptivo, comprensivo y crítico de los problemas sociales desde la psicología, donde cada vez más la subjetividad como concepto genérico se vislumbra como eje del análisis de lo que los sociólogos llaman "temas emergentes". Las problemáticas de desempleo, inseguridad, educación, gerenciamiento de organizaciones, trabajo en red, etc., tienen la cualidad de anudar los factores subjetivos (de pensamiento, sentimiento y acción) con las pertenencias grupales, institucionales y culturales. Estos temas emergentes se ponen sobre el tapete en polémicas cotidianas sobre la seguridad ciudadana y la delincuencia, la corrupción, la violencia intrafamiliar, la sexualidad y el VIH-SIDA y sobre la "superación de la pobreza".

Los grandes problemas que enfrenta la América mestiza, en este proceso de globalización, y que requieren del aporte de la ciencia, la academia, la profesión y la investigación psicosocial, serían entre otros: espacios urbanos y rurales, sustentabilidad, interculturalidad, derechos de minorías discriminadas, género, violencia social y doméstica, relación entre representación de ciudadanía y confianza en la democracia, adicciones diversas, exclusión y marginación social, etc. A las seculares condiciones de desigualdad, pobreza y exclusión que han acompañado la historia de América Latina, se han sumado nuevos e inéditos fenómenos que reproducen el circuito perverso de empobrecimiento material y espiritual de las personas y las sociedades. A los viejos pobres se les une ahora jóvenes precariamente empleados o excluidos con mayor frustración.

Se precisa ampliar la noción de los derechos humanos en el sentido de los derechos colectivos, como los de las mujeres e indígenas. La cuestión indígena es una de las luchas emancipatorias americanas, que nos va a convocar a pensar la autodeterminación de una nueva manera. La globalización involucra el reto hacia una política de la identidad y la diferencia. Dentro de la globalización, se están forjando nuevas identidades donde se conjugan diferentes regímenes históricos y epistémicos, donde el despertar de las tradiciones, la supervivencia de significados y la gestación de nuevos saberes, se articulan con las ciencias y tecnologías modernas; donde se abren las posibilidades para la convivencia de lo diverso.

Las intervenciones en comunidades y a nivel social, desde la perspectiva de la psicología social comunitaria, están orientadas por las nociones de cambio social a través de la concientización y subsiguiente participación las comunidades en la solución de sus problemas. Este proceso requiere que la comunidad acceda a recursos materiales y psicológicos, y adquiera a través de ellos un mayor control sobre su ambiente. Aquí se presenta el reto de superar el asistencialismo-paternalismo-clientelismo y desarrollar el compromiso con el cambio social con las mayorías, no sustituyéndolos se hace más evidente. Así, Amalio Blanco (1988) en La Psicología Comunitaria ¿Una nueva utopía para finales del siglo $X X$ ? refiere que esta definición de utopía puede venir por varios rasgos que la 
caracterizan, como: interés por la comunidad, modo de relación en contraposición con el individualismo, por la demanda de tolerancia, respeto y consideración por la diversidad y la diferencia, lo que significa también aceptar que pueden y deben coexistir valores y normas diferentes. Por la visión teórico-contextualista que concede a los factores y variables externos un papel decisivo, pero no exclusivo en la determinación del equilibrio y ajuste psicológico de las personas, con lo cual, lo coherente es intervenir sobre tales factores - provocar un cambio social- junto con la creencia de que cada individuo puede llegar a ser quien oriente y dirija su existencia y por la vocación preventiva de la Psicología Comunitaria.

La Psicología Comunitaria, al estar en estrecha relación con los conflictos humanos y aportar soluciones grupales en el área de la comunidad, tiene un fuerte contenido práctico y esto ha influido favorablemente en su desarrollo empírico en detrimento de la teoría. Sin embargo, esto no significa que no existan aportes en este sentido, sino que los diversos modelos teóricos resultan de las posturas científicas y filosóficas de sus creadores.

Bandura (1996) sigue siendo uno de los teóricos que ha hecho aportaciones más comprensivas acerca del papel que tiene la "autoeficacia percibida" en la conducta de las personas, ello nos llevó a trabajar con dicho enfoque, pero asumiéndolo desde la perspectiva de la autopercepción de actores que generan prácticas profesionales y académicas: los propios psicólogos sociales.

Habiéndose ubicado la diversidad de agendas para la psicología social nuestro objetivo consistió en identificar la autopercepción de logros que los psicólogos sociales tienen en relación con su ubicación geográfica (costa, sierra y selva) habiéndose evidenciado la poca presencia de psicólogos sociales en regiones como la selva y en menor medida en la sierra peruana, así como su dispersión en la zona costera, centralizándose su presencia principalmente en las grandes urbes (Lima, Arequipa, Trujillo, Chiclayo, Huancayo). En estos últimos ámbitos geográficos se encontró que se reconocían mayores logros frente a determinados problemas (principalmente de salud, educación, organización, y en menor medida, en aspectos macrosociales como la seguridad, corrupción, trabajo, desempleo, etc.) de un nivel microsocial o social-comunitario.

\section{Conceptualización de las variables estudiadas}

\section{Autopercepción de logro y eficacia}

En relación con la autopercepción de logro y eficacia hemos extraído los planteamientos de Bandura (1982), Catalá (1983), Barbara Marin (2003). Bandura (1996) define la "autoeficacia percibida" como: Logros anteriores; experiencia vicaria, persuasión o influencia recibida.

Unas mínimas expectativas de autoeficacia en torno a las propias capacidades constituyen un atributo importante en la evaluación del sujeto. Los profesionales opinan que una cierta percepción de autoeficacia tiene que ir acompañada del deseo de cambiar y reconocimiento del problema. 
La contribución de la psicología social a la prevención y disminución de problemas de salud ha sido abordada por Marín (1983). Por ejemplo, en los pacientes de cáncer se puede intervenir para mejorar su percepción de control.

Catalá (2003) trata de aprehender los factores psicosociales que intervienen tanto en los procesos de adicción, como en los de cambio de estilos de vida.

Así, las creencias que cada individuo tenga sobre sus capacidades es un factor de primer orden para cualquier solución de problemáticas.

Lévy-Strauss (1958) mostraba en sus obras que las diferentes culturas de los seres humanos, sus conductas, esquemas lingüísticos y mitos revelan la existencia de patrones comunes a toda la vida humana. Lo cual nos debe llevar a discriminar qué aspectos favorables a una mejor calidad de vida en otros países pueden ser aplicados a nuestra realidad.

En el campo ambiental, los planteamientos de organismos internacionales como UNESCO, Cumbre sobre el Medio Ambiente o Cumbre de Río, Programa Europeo, Protocolo de Kyoto, etc., han puesto de relieve el comportamiento humano como factor fundamental en entornos urbanos, rurales, empresa, etc.

\section{Responsabilidad social}

Las nuevas amenazas tecnológicas sobre el medio ambiente y el estatus biológico del ser humano, en el nuevo contexto económico, ideológico y político instaurado en los ochenta, promueve un nuevo compromiso colectivo en contra de la tendencia liberal de los sesenta orientado al individualismo. La figura dominante del individualismo democrático fue durante tiempo la euforia liberadora, y ahora es, cada vez más, la dificultad para vivir, la inseguridad, el miedo ligado no solo al terrorismo sino a la alimentación, lo relacional, la edad, el trabajo, la jubilación.

En síntesis, es sencillo comprender el porqué prosiguen las furias consumistas. Según Lipovetsky (2003), hemos pasado a la tercera parte de la historia de la moral, que denomina posmoralista, la cual rompe el proceso de secularización establecido a finales de los siglo XVII y XVIII. Sociedad posmoralista que no significa sociedad posmoral, sino sociedad que exalta los deseos, el ego, el bienestar individual en mayor medida que la abnegación. El ámbito de la sexualidad goza de plena libertad; el suicidio ya no se le considera una trasgresión a una obligación moral superior. El renacer ideológico de la familia no significa, en modo alguno, la rehabilitación de los deberes familiares, es decir, la sumisión del individuo a los deberes para su colectividad que representa la familia, sino la promoción de una familia psicologizada, que se rige según el principio de la autonomía individualista.

Zaragoza (2000) la define así: es la capacidad y obligación de responder ante la sociedad como un todo, por acciones u omisiones y que se ejerce, cuando corresponde desde alguna persona hacia todas las otras. La capacidad de responder está dada por los recursos con que cuenta la persona o el colectivo.

Berman (1997) la define como el compromiso personal de cada sujeto con las demás e incluye: entender que cada persona pertenece a una red social más amplia que tiene 
influencia decisiva en la construcción de la propia identidad; relaciones interpersonales basadas en éticas de justicia y preocupación por otros y; actuar con integridad en relación a los valores.

\section{Representaciones sociales}

Autores como Moscovici (1965), Baro (1982), Jodelet (1989), Parker (1988) han dado significativos aportes.

Moscovici (1984) la define como: modalidades particulares del conocimiento, cuya función es la configuración de los comportamientos y la comunicación entre los individuos. Baró (1982) dice: "Una sociedad mantiene su unidad debido a la existencia de una conciencia colectiva. La conciencia colectiva consiste en un saber normativo, común a los miembros de una sociedad".

En relación con el siguiente objetivo, describir la representación de eficacia social de los programas de investigación y profesionales a nivel de las políticas, programas y estrategias sociales, encontramos evidencias que permiten afirmar que se generó interés de instituciones en la comprensión desde la perspectiva psicosocial y en programas de capacitación y formativos sobre temas de violencia, participación social, etc., así como la inserción de los psicólogos sociales en equipos multidisciplinarios.

Se han introducido temas psicosociales en las agendas regionales: violencia intrafamiliar, estrés postraumático psicosocial, corrupción, pobreza, desarrollo, problemas educativos, calidad de vida (laboral, sexual, materno-infantil).

Se asume que existen algunos programas psicosociales eficaces en diversos ámbitos de intervención: maltrato familiar en DEMUNAS, prevención de violencia juvenil y escolar, barrera de contención de los problemas de sexualidad, la prevención del deterioro ambiental con limitaciones -en la gran minería y la minería artesanal- al desarrollo de la ciudadanía y democracia. En general, los psicólogos sociales demuestran alta responsabilidad social a través de sus compromisos con agendas establecidas, trazándose metas basadas en la prevención de aparición de problemas futuros.

\section{MÉTODO}

El diseño empleado es de aproximación holística, cualitativa y sistémica.

Se seleccionaron como sujetos participantes:

a. Profesionales en ejercicio ubicados en Facultades de Psicología de diversos lugares del país que pertenecen a instituciones y desarrollan políticas y programas.

b. Informantes calificados, operadores de las políticas o programas con contenido psicosocial.

c. Las propias políticas y programas de formación y aplicación psicosocial.

Las técnicas de recolección de información fueron: a) entrevistas a profundidad, b) grupos focales, c) análisis de contenido y hermenéutica. Se usaron técnicas de triangulación. 
Se ha considerado la metodología cualitativa como la más apropiada para el logro de los objetivos propuestos, pues con estas técnicas obtenemos datos descriptivos, basados en las propias palabras de las personas con las que tratamos de obtener una comprensión detallada en torno al proceso que incluye su acción como su autopercepción; mientras que el análisis documental sirvió para las políticas y programas de intervención psicosocial.

No hemos recurrido a la técnica desarrollada por Iñiguez, pero se han podido constatar marginalmente que en nuestro país es factible seguir esa perspectiva para contestar preguntas importantes que se señalan más adelante. Hemos preferido "mapear exploratoriamente" a los principales profesionales de la psicología social en el Perú y del mismo modo ubicar la acción de estos en los programas y políticas de diversas instituciones de gestión social, tanto públicas como de la propia sociedad civil en algunas de las principales regiones del Perú.

Es en la perspectiva de Iñiguez que algunas preguntas podrían ser respondidas con relativa facilidad analizando los congresos y otros tipos de reuniones científicas. En el caso de la psicología social: ¿Podemos identificar a los “líderes” de la psicología social y sus grupos? ¿Cuáles son sus subdisciplinas, sus áreas de investigación, sus tópicos de interés, etc., ¿Cómo se relacionan estas subdisciplinas, áreas tópicos y/o orientaciones las unas con otras? y ¿se puede cartografiar la dinámica disciplinaria de la psicología social? ¿Podemos determinar cómo y cuándo áreas de investigación periféricas con sus propios programas de investigación se unen a la corriente central de la psicología social?

El esquema de las entrevistas a profundidad usado tuvo en consideración: En un primer momento lanzamos una pregunta amplia: Desde tu punto de vista, como psicólogo que has tenido contacto y has acompañado a algunos programas de intervención, ¿podrías decirnos qué hace posible, qué es necesario y cómo un conjunto de acciones pueden aportar con efectividad en el desarrollo humano pleno? Para luego profundizar indagando otros aspectos relacionados.

Durante los encuentros tratamos que se aborden los siguientes temas que consideramos de interés, ya sea de manera espontánea o preguntando sobre los mismos. La segunda entrevista nos sirvió para las aclaraciones, tanto del entrevistador como del entrevistado, y para profundizar en los temas abordados o plantear nuevos, si se quedaron pendientes. Los aspectos a indagar los hemos dividido así:

Personales: Motivación o deseos de cambio, sensación de autoeficacia, autoestima, responsabilidad individual o social. Ambientales: La familia, apoyo social, redes sociales, tipos de estrategias, grupo de iguales, autoayuda o asistencial. Institucionales: La política incluye estrategias o teorías psicosociales, los programas se desarrollan dentro de estos parámetros, teorías psicosociales que se explicitan.

\section{Clasificación y reorganización del material}

La realizamos siguiendo el Esquema de Deslauriers (1985), modelo mixto que parte de que algunas categorías son preexistentes en el inicio del contenido (elaboraremos un guión de temas objeto de estudio y aspectos de interés), pero sin cerrar la posibilidad de ir 
incorporando nuevas categorías y reemplazando o modificando las prefijadas. El análisis minucioso de todo el material recogido se hizo inmediato a la transcripción, lo cual permite ir acumulando conocimiento a medida que se desarrolla el estudio.

Se generó interés de las instituciones en la comprensión desde la perspectiva psicosocial y en los programas de capacitación y desarrollo de programas formativos.

Habiéndose ubicado la diversidad de agendas para la psicología social, nuestro objetivo fue identificar la autopercepción de logros de psicólogos sociales en costa, sierra y selva. Encontrándose concentración en las grandes urbes donde se reconocía mayores logros en salud, educación y organización y en menor medida en seguridad, corrupción, trabajo, desempleo.

En cuanto al segundo objetivo planteado en nuestra investigación, que fue describir la representación de eficacia social de los programas de investigación y profesionales, se asume que hay programas eficaces en diversos ámbitos de intervención: maltrato, violencia juvenil, problemas de sexualidad, ambientales y democracia.

De este modo se ha respondido la pregunta de ¿Cómo perciben y representan la eficacia de su quehacer los psicólogos sociales y cual es la relación con el nivel de representación social que ellos han ido construyendo en el decurso de su formación académica y profesional?

\section{RESULTADOS}

Se demostró la efectividad de la aplicación de la psicología social en políticas y programas sociales frente a los problemas prioritarios, es decir, la agenda, construyendo indicadores para facilitar efectividad de los programas en lo formativo, profesional e investigativo y teórico.

Resultados más relevantes:

Se generó interés de las instituciones en la comprensión desde la perspectiva psicosocial y en programas de capacitación y desarrollo de programas formativos: sobre temas de violencia, de participación social, etc. Inserción de los psicólogos sociales en equipos multidisciplinarios. Introducción de temas psicosociales en las agendas regionales: violencia intrafamiliar, estrés postraumático psicosocial, corrupción, pobreza, desarrollo, problemas educativos, calidad de vida (laboral, sexual, materno-infantil).

Existen algunos programas psicosociales eficaces en diversos ámbitos de intervención: maltrato familiar en las DEMUNAS, prevención de la violencia juvenil y escolar, barrera de contención de los problemas de sexualidad, la prevención del deterioro ambiental con limitaciones -en grandes minas y minería artesanal, al desarrollo de la ciudadanía y democracia-. En general los psicólogos sociales demuestran alta responsabilidad social a través de sus compromisos con agendas establecidas trazándose metas basadas en la aparición de problemas futuros.

Para poder ver la panorámica de interés por temas psicológicos, muestreamos los temas de los proyectos de investigación del Instituto de Investigaciones Psicológicas (IIPSI) en los últimos cinco años y los Artículos de Investigaciones en revistas del IIPSI (ver tablas). 


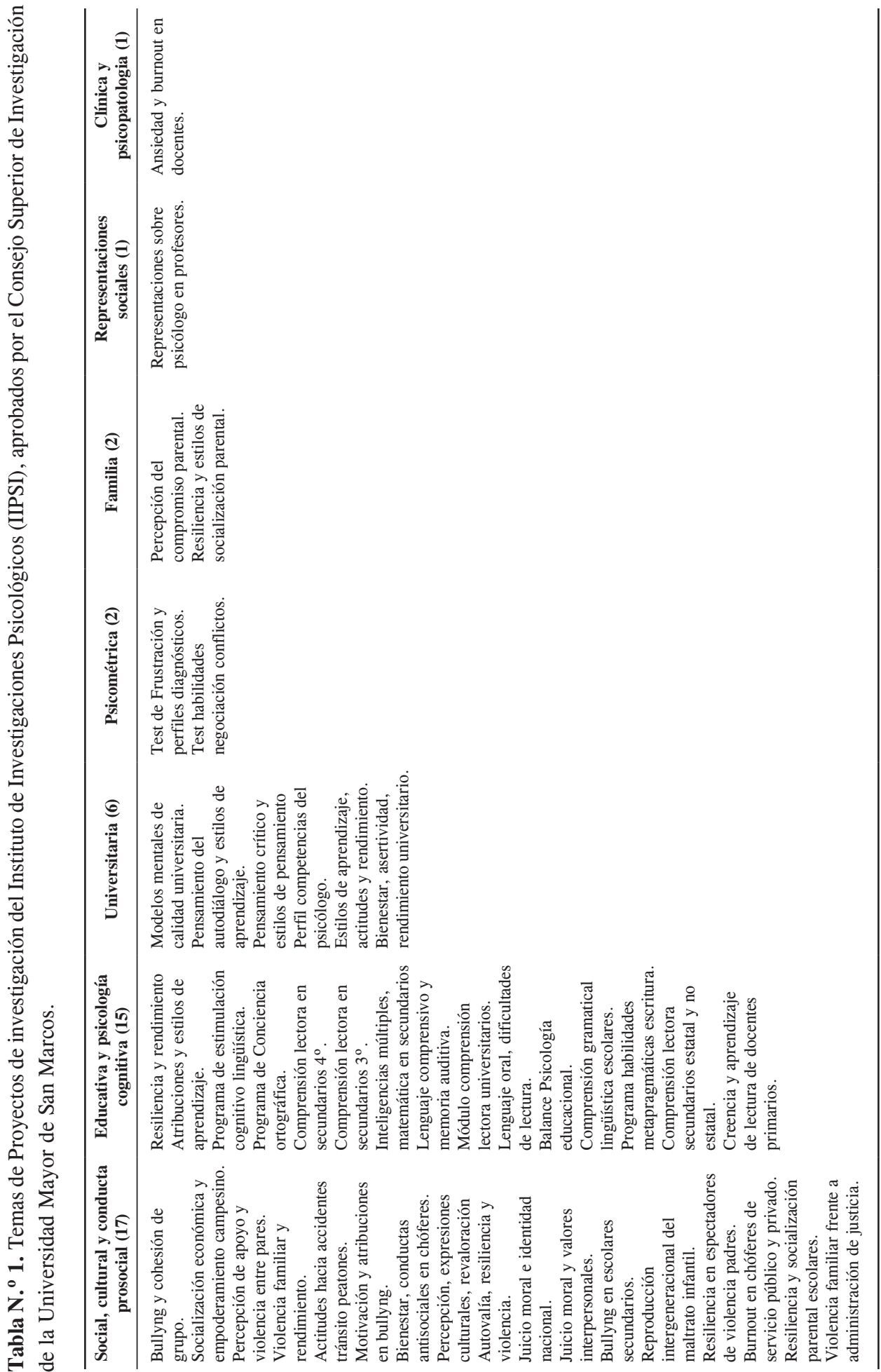


Como podemos apreciar el interés por los temas psicosociales es muy notorio dentro de las investigaciones presentadas al Instituto de Investigaciones Psicológicas de San Marcos que junto con los temas de psicología escolar son los más frecuentes.

Igualmente, podemos apreciar que el interés por los temas psicosociales es muy notorio dentro de las publicaciones de la Revista del IIPSI, aunque ocupan el segundo lugar junto con los temas universitarios, el primer lugar lo ocupan los temas de psicología escolar.

Propuestas de agenda psicosocial:

Violencia, maltrato, estrés postraumático, efectos de la pobreza en el desarrollo psicológico, salud mental dentro del campo de la salud pública, problemas de sexualidad temprana, deterioro ambiental, derechos humanos, desarrollo de ciudadanía, participación social y contaminación ambiental, etc.

Se logra identificar la autopercepción de los logros que los propios psicólogos sociales tienen de su profesión, en principio de manera muy difusa a nivel general, cuando se ubicaron a grupos de psicólogos sociales principalmente en Lima Metropolitana, estos demostraban un alto nivel de conciencia de los logros de que su disciplina está aportando al bienestar y desarrollo humanos.

Se evidenció la inserción de los psicólogos sociales en equipos multidisciplinarios y en agendas regionales: violencia intrafamiliar, estrés postraumático psicosocial, corrupción, pobreza, desarrollo, problemas educativos, calidad de vida, laboral, sexual y materno infantil.

Se asume la existencia de programas psicosociales eficaces en diversos ámbitos: maltrato familiar (DEMUNAS), prevención de violencia (Educación), prevención en problemas sexualidad, ambientales (regiones de grandes asientos mineros y minería artesanal), ciudadanía y democracia.

En general, los psicólogos sociales demuestran alta responsabilidad social. A través de sus compromisos con agendas establecidas, trazándose metas basadas en la aparición de problemas futuros.

\section{DISCUSIÓN}

De los resultados tentativos se pueden colegir una serie de explicaciones plausibles que requieren aún profundización.

1. La autopercepción de logros (teóricos) se confunde con la autopercepción de eficacia.

2. Se debe incluir aspectos no solo de índole cognitivo sino también afectivo, como: autoevaluación, autoestima, sentimiento de eficacia.

3. Se requiere profundizar en conocer el desarrollo desde los eventos nacionales y programas en los que se descubra la existencia de comunidades y redes constituyendo redes sociales y comunidades científicas y profesionales.

4. Existe dificultad en identificar a los profesionales que desarrollan el campo psicosocial en el país, debido a la poca producción y difusión de sus obras y acciones, y a la poca visibilidad pública. 


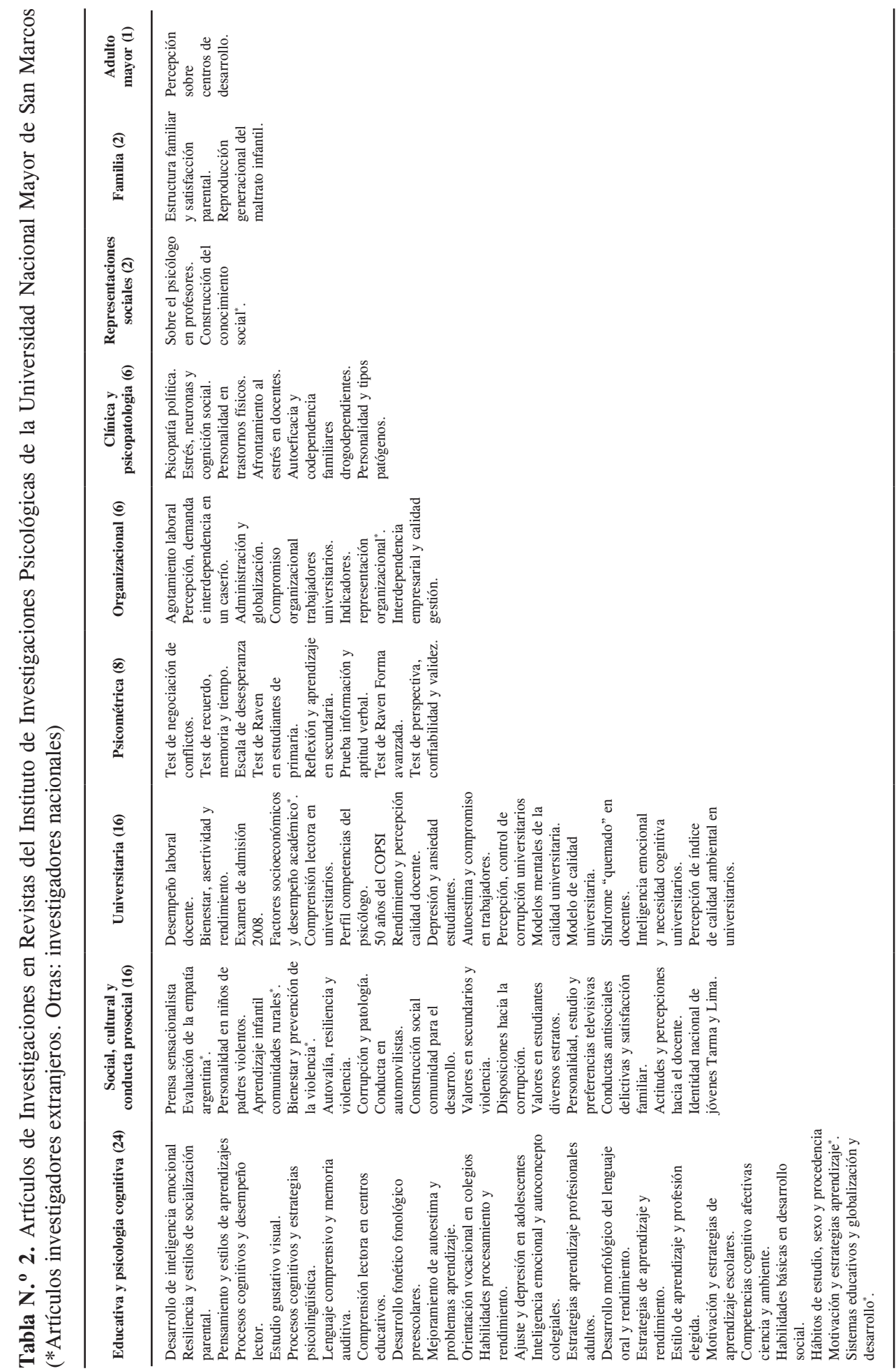


5. En relación a lo anterior, desarrollar entrevistas a profundidad se constituye en una técnica de suma importancia, pero también limitante para abarcar grandes conjuntos de psicólogos sociales en el país.

\section{CONCLUSIONES}

1. La presente investigación ha tenido otros abordajes como los de Orellana et al. (2008) con la investigación: "Representaciones sociales sobre el psicólogo en profesores de educación básica pública y privada de Lima (costa) y provincias (sierra y selva). En ella se coincide en diversos aspectos como que las representaciones sociales son diferenciadas por regiones y se precisa que se expresa en la costa como: investigador especializado, psicoterapeuta y consejero; en la sierra: concientizador y promotor de bienestar y en la selva: promotor de felicidad y de ética. Señala que los psicólogos clínicos y educativos sean reconocidos en costa y sierra, pero en la selva el psicólogo es único.

2. La autopercepción de logros tiene relación con su ubicación en costa, sierra o selva, diferencialmente. En la selva: ausencia de programas formativos y fragilidad institucional, el papel de la Psicología Social está restringido. Temáticas: suicidio, resentimiento contra el Estado por la exclusión que sufren, identidad, ecoeficiencia en los sectores productivos y protección del ecosistema. En la sierra: Presencia restringida a zonas de violencia política, con programas de estudio e intervención ante estrés postraumático. En la costa: centros formativos con limitaciones en la especialización algunos avances: maltrato, niños en riesgo, adolescentes, sexualidad, jóvenes, violencia. Algunos programas de desarrollo.

3. La divulgación de aportes de la psicología social en la sociedad podría tener un impacto en el mejoramiento de calidad de vida, bienestar y del desarrollo humano en nuestro país.

4. La generación de indicadores de evaluación de la efectividad y eficiencia, tanto desde la perspectiva profesional, académica e investigativa permitirá que se avance en la acreditación de la psicología y el reconocimiento de la responsabilidad social e institucional de la psicología.

\section{NOTA DE RECONOCIMIENTO}

Los autores reconocen la colaboración de los siguientes investigadores: César Cuya, Isabel Ale, David Tarazona y Marco Peña.

\section{REFERENCIAS BIBLIOGRÁFICAS}

1. Albiach, C. (2003). El proceso de cambio en la adicción a la heroína, un análisis a través de metodologías cualitativas. Universitat de Valencia: Server de publicacions,

2. Arenas C. (2006). La evaluación docente: una mirada desde la psicología social, responsabilidad social, formación docente y retos de la educación. En prensa. 
3. Clemente M. (1997): Psicología social aplicada. Madrid: Pirámide.

4. Iñiguez L.; Justicia J.; Peñaranda M.; Martínez L. (2006): La psicología social en España: estructuras de comunidades. En: Redes, Revista hispana para el análisis de redes sociales. Vol. 10 (3).

5. Levy Strauss C. (2006). Antropología estructural, mito y sociedad, $14^{\mathrm{a}}$. edición, México: Casa del libro.

6. Lipovetsky, G. (2003). Metamorfosis de la cultura liberal. Barcelona: Anagrama.

7. Marín, B. (1983). El uso de la psicología social en la promoción de la salud. $\mathrm{Ph}$. D. Health Psychology Program. San Francisco: University of California, Diciembre No 12, 1983 Papeles del psicólogo.

8. Montgomery, W. (2005): El quehacer conductista hoy. Ensayos de interpretación teórica y práctica. Lima: Ed. RPFA.

9. Zaragoza, F. (2000): Cultura de paz. Conferencia dictada en las XIX Jornadas Interdisciplinares: La responsabilidad social en la Educación. Documento: http://www.ua-ambit.org/jornades2000/Ponencias/joo-mayor-zaragoza.htm 